\title{
'Mini-interval gait' switching: understanding the positive implications of a novel training regime
}

\author{
James L. Croft ${ }^{1, *}$, John E. A. Bertram ${ }^{1,2,3}$ \\ ${ }^{1}$ Centre of Exercise and Sports Science Research, School of Medical and Health Sciences, Edith Cowan University, Perth, Australia \\ ${ }^{2}$ Biomedical Engineering Graduate Program, University of Calgary, Calgary, Canada \\ ${ }^{3}$ Cumming School of Medicine, University of Calgary, Calgary, Canada
}

The neuromechanical reorganization required to change gaits imposes an energetic cost $75 \%$ greater than either a walking or running step at the same speed. By combining walking and running with the requisite gait switching transition steps, an exercise protocol can be generated with virtually any desired metabolic output even at relatively slow treadmill speed. Gait switching increases metabolic demand through discrete events, which can be tolerated more easily by individuals recovering from health problems, just as interval training allows greater work production for healthy individuals. In addition to cardio-respiratory benefits, 'mini-intervals' with frequent gait switching also provides positive effects and attributes such as distributing muscle group activation, re- training neural coordination, and avoiding repetitive joint overloading. It has the added benefit of developing stability during transitions while a safety hand rail is present which can lead to greater stability in more complex natural environments. Finally, increased mental focus may help avoid the monotony of usual treadmill workouts, aiding adherence to an exercise program. We review evidence for the cost increase of the gait transition step and explain the mechanisms involved. We also discuss literature supporting the range of benefits for mini-interval gait switching as a training and rehabilitation tool.

Keywords: Walking, Running, Transition, Intervals, Health, Treadmill

\section{INTRODUCTION}

The treadmill is an effective, convenient and consistent device for increasing activity. Vulnerable populations like those who are ambulatory but recovering from injury or illness, or those beginning a fitness program following limited activity may benefit from the consistent surface and available handrails. Treadmill exercise also has drawbacks, such as the notoriously hard surface, the relatively high speeds required to burn substantial calories or influence cardiopulmonary health and blood lipid levels (Duncan et al., 2005), the monotonous environment, and lack of neuromuscular adaptability to features that are found in overground locomotion. An effective treadmill-based protocol that mitigated these drawbacks and provided a complete and effective activity program would be of value. This would be particularly useful if the protocol were appropriate for populations that are capable of moderate activity but might be physically vulnerable early in their training program. Although there are some small differences in kinematics (Alton et al., 1998; Hollman et al., 2016; Lee and Hidler, 2008) and ground reaction force (White et al., 1998) between treadmill and overground walking, the differences are minor (for example, a ground reaction force with approximately $98 \%$ correlation), and the benefits from treadmill training in this population would be readily transferred to overground locomotion.

Interestingly, the patient base we describe often spontaneously adopts a novel movement regime that we suspect maximizes treadmill effectiveness. When beginning a treadmill training regime many individuals will frequently switch between walking and running gaits at the same, low or moderate speed. This 'mini-interval' training strategy has a number of advantages over training with a constant gait, and we suspect that these are under appreciated. Mini-interval training would work just as well for overground
${ }^{*}$ Corresponding author: James L. Croft (iD https://orcid.org/0000-0002-7457-0837 Centre of Exercise and Sports Science Research, School of Medical and Health Sciences, Edith Cowan University, 270 Joondalup Drive Joondalup Western Australia 6027, Australia

E-mail: j.croft@ecu.edu.au

Received: April 8, 2019 / Accepted: May 29, 2019
This is an Open Access article distributed under the terms of the Creative Commons Attribution Non-Commercial License (http://creativecommons.org/licenses/by-nc/4.0/) which permits unrestricted non-commercial use, distribution, and reproduction in any medium, provided the original work is properly cited. 
locomotion but it would be more challenging to determine energetic cost without a means to control speed. In this contribution we analyze the benefits that can arise from using two mechanically distinct gaits, combined with the process of active switching between them.

\section{GAIT: UNDERSTANDING THE DYNAMICS OF LOCOMOTION}

The human walk has been well described, but still remains relatively poorly understood. Classic descriptions of Saunders et al. (1953) and Inman et al. (1981) identified six determinants, or influential components, of the walking gait. Some of these determine the fundamental motions involved (motion of mass as it is supported by the legs in the compass gait), others are seen as modifiers that smooth the centre of mass $(\mathrm{CoM})$ path (pelvic tilt, rotation, and stance leg flexion), while ankle plantar flexion near the end of foot contact establishes leg swing velocity.

Electronic force platforms allowed recognition that whole body kinetic and potential energy were largely out of phase during the walking cycle, reminiscent of a swinging pendulum, albeit an inverted pendulum where the mass of the body moves in an arc on the extended limb. Exchange between these two energy forms has been interpreted as a main reason that the normal walking gait is effective. The passive (or near passive) exchange means that the energy of subsequent steps need not be regenerated for each new step, but can be recovered from the previous step (Cavagna et al., 1976).

However, both walking and running do require energetic investment - not all energy is available for reuse. Recent studies (some arising from designing energetically effective bipedal robots: Kuo, 2007; Srinivasan, 2011; Srinivasan and Ruina, 2006) suggests that insight can be gained by tracking mechanical energy that leaves the system (how energy is lost, rather than how it shifts and is recovered within the system).

In considering walking or running it is important to define the task itself. It is common to consider the task of gait as all of the actions required to produce the motions observed. Such definition includes both the task goal (the problem) and the movement strategy utilized to accomplish the goal (the solution) (Croft et al., 2017). Separating the task from the mechanisms employed to accomplish it puts the mechanisms in their functional context, so their contribution (and limitations) can be more readily identified. What is the fundamental task of walking and running and what mechanisms are employed to accomplish that task?
The fundamental task of walking and running is to manage the interaction of the individual's mass with the supporting surface. This interaction is managed by the action of the legs and the process results in the gaits we can identify (which are common because they are effective). We use this perspective to consider the similarities and differences between walking and running.

The task is similar for walking and running but the differences we perceive are due to different movement strategies employed to satisfy the task. This results in different energetic cost structures. Walking is cost effective at slow speeds but energetic cost rises sharply as speed increases. In running the energetic cost per distance covered is relatively insensitive to speed (the rate of energy use, power, does increase with speed but at faster speeds the stride length increases so energetic cost per distance remains fairly constant over normal running speeds). Different neuromuscular strategies are employed for each gait, providing an opportunity to exploit the difference to benefit the individual.

Walking and running both have a low-cost and a high-cost portion of the gait cycle, determined largely by the change in the vertical direction of the CoM. During single stance in walking or noncontact in running the $\mathrm{CoM}$ reaches its highest point, and then descends prior to contact of the next stance leg. This transition from upward to downward trajectory is driven passively by gravity, so involves little energetic cost (the inverted pendulum of walking and ballistic flight of running). The opposite direction change, moving the CoM upward from its downward fall must be actively achieved via action of the leg(s). This occurs during double stance in walking and stance in running. A main objective of both gaits is to manage this downward to upward transition as effectively as possible. Different action strategies are available in the two gaits, simply because this transition occurs with two legs in walking, and only one in running (Bertram and Hasaneini, 2013). This is the basis of the differences in neuromuscular activity between walking and running, and forms the foundation for our contention that the two gaits are two distinct activities. As a consequence, an activity session sharing both has advantages over either alone.

\section{GAIT CHANGE}

The change from one gait to another has to be actively generated and is indicated by $75 \%$ greater metabolic cost over either a walking or running step at the same speed (Fig. 1; Usherwood and Bertram, 2003). Spontaneous gait transitions, whether from a walk to run or a run to walk, involve sudden changes in several 


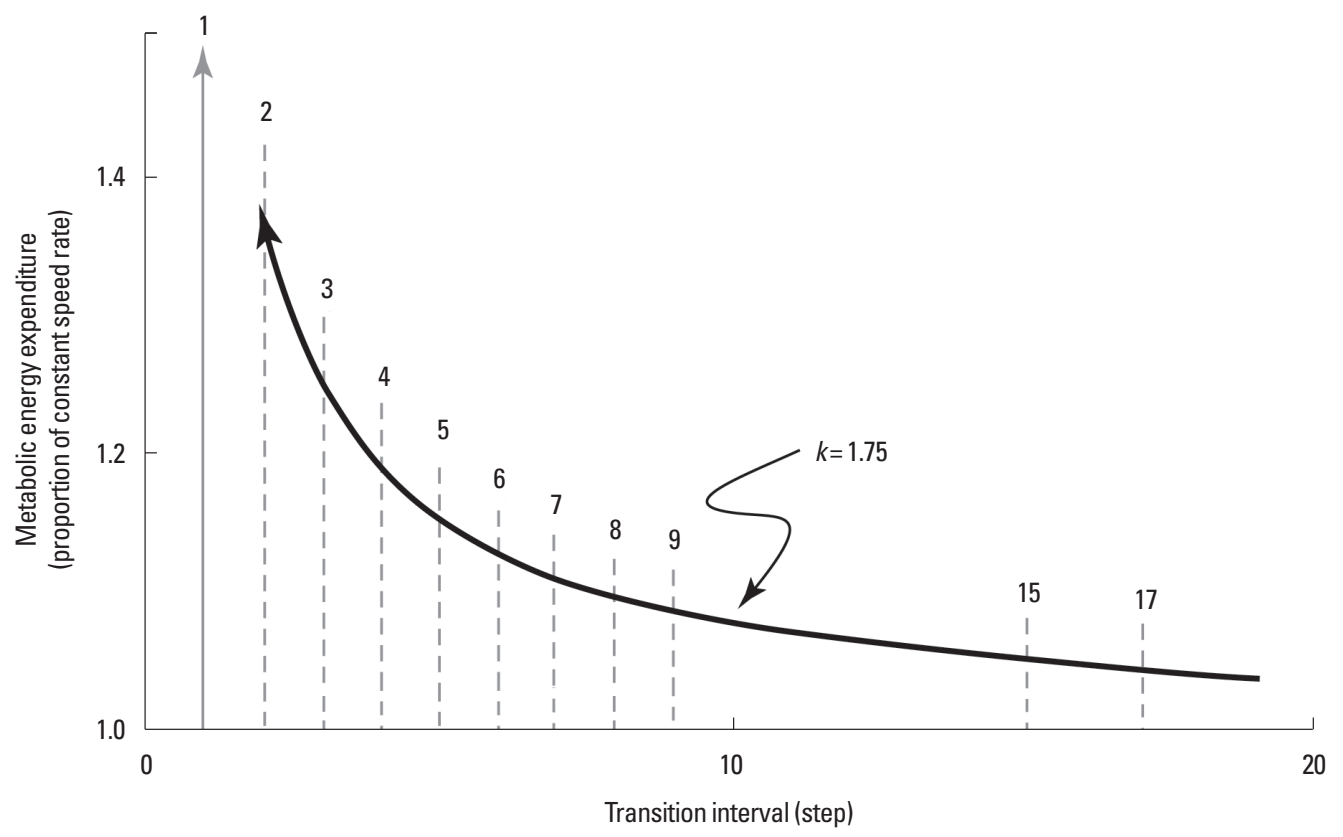

Fig. 1. The relationship between the rate of metabolic energy expenditure and the number of steps between gait transitions (transition interval $T 1$, after 10). The relationship was constructed using subjects who transitioned between gait after a specified transition interval (intervals used indicated by vertical dashed lines with number for the interval used). Subjects were not able to switch gait with each step $(T I=1)$ but the metabolic cost of a single transition could be extrapolated from the relationship: $k=T /\left\{\left[\left(P_{x}+P_{\text {trans }}\right) / P_{x}\right]-[1-(1 / T I)]\right\}$, where $P_{x}=$ metabolic power required for steady speed movement at the speed where walking and running metabolic power are equal (approximately $2.1 \mathrm{~m} / \mathrm{sec}$ ), $P_{\text {trans }}=$ metabolic power of the transition step and $k=1.75$ (the experimentally determined cost of a transition step). It is possible to use this relationship to estimate the increase in metabolic energy expenditure for a given transition interval. For instance, a T/ of 10 increases the cost of moving on a treadmill by approximately $7.5 \%$ while a $T /$ of 5 increases it by approximately $15 \%$.

spatiotemporal variables (Segers et al., 2013), e.g., the phase relationship of potential and kinetic energy, foot strike and leg orientation as well as trunk lean, action of the last half of leg swing and the power transferred across the leg joints. These factors indicate that the transition step has substantial differences from either a normal walk or run step. Although the transition occurs within a single step, transition influences can be detected in either the preceding step in the walk-to-run transition, or the following step in the run-to-walk transition (Segers et al., 2007).

\section{MINI-INTERVAL GAIT SWITCHING AS A TRAINING STRATEGY}

Using brief walking and running intervals (with frequent transitions) may have many advantages over treadmill training involving one gait alone: (a) increased cardio-respiratory performance; (b) muscular activity by increasing the number of muscle groups activated; (c) adapting neuromuscular control and coordination; and, (d) shifting joint load pattern. Gait switching at short intervals can increase the overall effectiveness of the exercise while protecting against overuse injuries.

\section{CARDIO-RESPIRATORY BENEFITS}

Interval training improves cardiovascular fitness more than moderate continuous exercise in populations ranging from athletes (Helgerud et al., 2007) to patients post cardio-infarction (Wisløff et al., 2007). In fit individuals running may be required to sufficiently raise heart rate. In less fit individuals walking uphill at moderate speed may be sufficient but there may be extra benefits of including moderate speed running in the protocol. Short duration bouts of walking and running with intervening gait transitions provides a simple but comprehensive exercise protocol that increases general fitness that prepares individuals for successful real-world ambulation within a safe and controlled situation.

Walking is considered a lower speed gait than running but the range of speeds where running is possible is actually broader than for walking. Running is possible from zero speed (running in place) through to the individual's maximum speed. The dynamics of walking require falling forward, however, so a true functional walk requires moving forward (marching in place moves the arms and legs, but does not involve the ground reaction forces, contact 


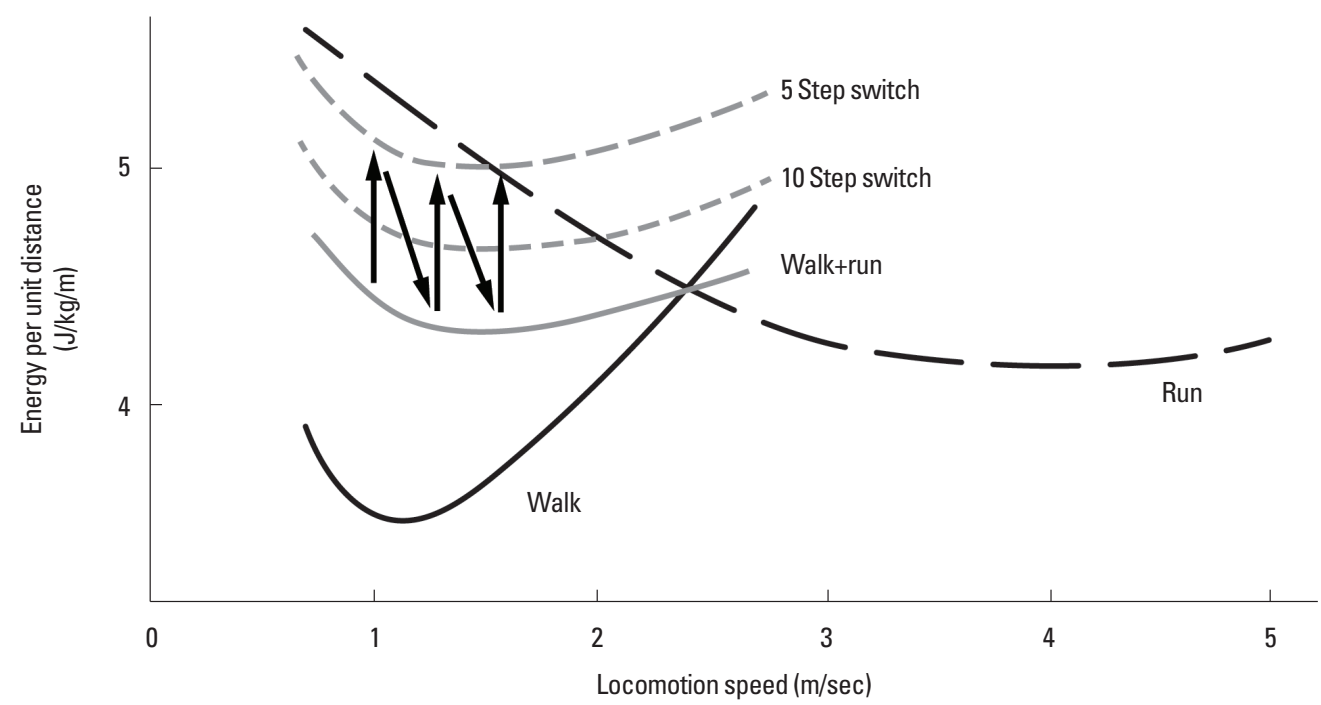

Fig. 2. Cost of transport per unit distance for walking and running normalized per kg body weight (redrawn from Long and Srinivasan, 2013). An estimate of the metabolic cost of running and walking for an average individual with no switching, and employing the mini-interval strategy with a switch every 5 steps, and every 10 steps is also shown.

dynamics, coordinated actions or energy exchanges that are characteristic of true walking). As speed increases to the natural gait transition and beyond, metabolic cost of walking increases sharply (Fig. 2; Sentija and Markovic, 2009) but it becomes impossible to walk much faster than a moderate run (Usherwood, 2005). Consequently, it is possible to run (jog) at any speed an individual finds comfortable for walking.

Walking is a metabolically effective gait at slower speeds and the cost of transport of walking is less than that of running except at higher speeds (Fig. 2) while running at low speeds is metabolically more demanding than walking. The metabolic cost surface likely dictates the selection of gait (walk or run) and the specific parameters, such as stride length and stride rate (Croft et al., 2019). Prilutsky and Gregor (2001) suggested that the sense of effort in the legs may be due to large activation of tibialis anterior, rectus femoris, and hamstrings during swing phase required during fast walking, and likely comes from motor commands rather than afferent force feedback. Alternatively, it has been suggested that walking and running depend primarily on different metabolite sources, where the perception of running and walking intensity depend on specific signaling from the body that encourages a more dependable energy source when locomotion intensities increase (Ganley et al., 2011).

Switching between gaits involves increased energy expenditure, so it is possible to increase overall metabolic demand by adding gait transitions, regardless of treadmill speed. However, this increased metabolic cost occurs in isolated events, that can be toler- ated more easily by individuals with limited cardiovascular endurance. The increased energetic cost of slow running also adds metabolic demand. Even at quite slow speeds using a walk and run with frequent transitions results in a substantial increase in energetic expenditure (Fig. 2).

Although for individuals with low activity levels any exercise is superior to none, only at higher intensity levels does exercise have a significant effect on cardiovascular function and blood chemistry (maximum $\mathrm{O}_{2}$ consumption, high-density lipoprotein [HDL] level, and HDL/cholesteroltotal ratio; Duncan et al., 2005). Gait transition increases metabolic activity at a discrete point, so does not require sustained higher power output. This allows an individual to perform greater overall cardiovascular work - in much the same way that standard interval training allows greater total work than sustained activity (Laursen and Jenkins, 2002; Tjønna et al., 2008).

\section{MUSCLE ACTIVATION BENEFITS}

As distinct gaits it is not surprising that walking and running have distinctive muscle activity patterns even at the same speed (Cappellini et al., 2006). This is partly due to the coordination strategy of each (see discussion above) and the difference in leg use strategy. An example is seen at the metabolic cost cross-over speed for walking and running (approximately $2.25 \mathrm{~m} / \mathrm{sec}$ for an average individual; Usherwood and Bertram, 2003). Walking at this speed is extremely demanding even though running is trivial for a healthy 
individual, in spite of the whole body metabolic demand being identical for both gaits. This could only result if substantially different muscularly driven activation strategies were employed in each gait.

The advantage to transitioning between gaits, then, means that different muscles will be activated. Besides providing relief from constant use fatigue, this also alters the load distribution within the muscles and involves a broad range of muscles in the total activity. Added to this are the muscles involved with active gait switching - the phase relationship of the arms, legs and trunk must be actively reorganized (Segers et al., 2013).

\section{NEUROMUSCULAR CONTROL AND COORDINATION BENEFITS}

Different muscle activation means changes in limb and body coordination (Diedrich and Warren, 1995). During the transition step the trunk is 20 degrees more anteverted compared to normal walking (Segers et al., 2013), requiring coordination between limb movement and body core support musculature. Decreased braking during the transition step also leads to a larger net propulsive impulse compared with the preceding and following steps (Segers et al., 2013). As such, walking and running with gait change at intervals will involve a much more complete set of muscle synergies than either walking or running alone.

It is beneficial to involve different motor groups or activity patterns in training (Dromerick et al., 2006), so an exercise routine involving both walking and running, even at a constant speed, will mean that both motor coordination patterns are practiced. As well, the gait change increases the complexity of the activity, developing and reinforcing motor synergies (Hagio et al., 2015) and neural adaptation (Kantak et al., 2012).

Although the gait transition occurs spontaneously when speeding up or slowing down, it appears to involve substantial cognitive coordination. For instance, subjects delayed the transition from walk to run or run to walk for gradual treadmill speed changes when confronted with a concurrent counting task (Daniels and Newell, 2003).

\section{CHANGES IN JOINT LOADING}

Although muscles activated during walking and running are similar, their timing, coordination and magnitudes differ (Abdolvahab, 2015). This results in different loading (magnitude, pattern and rate) and function of the leg joints between walking and running, even at the same speed. Walking involves longer strides but lower stride frequency than running at a given speed (walking strides are 20\% longer at 20\% lower frequency than running at the natural gait change speed [Pandy and Andriacchi, 2010]). The ground reaction profile of walking and running differ substantially, with the walking vertical force having maxima near both the beginning and end of foot contact while running is unimodal with the maximum occurring near mid-stance. Over the course of contact joint moments differ between walking and running, especially at the knee. Joint power is similar at the ankle for walking and running, but magnitudes are high for the knee and hip in running. Work across each joint differs between stance and swing, as well as between gaits (except for swing phase in the knee; Pires et al., 2014). Running, even at the same speed as a walk, involves more negative work (work on the joint by the external environment) at the knee but more swing leg work at the hip. More positive and negative work occurs at the ankle in a run than a walk (Pires et al., 2014). These differences occur due to the different muscles and activity levels involved with each gait. Joints do appear to require some loading to maintain cartilage health, even though overuse and overloading can cause damage leading to degenerative joint disease. Employing both gaits with frequent switching relieves consistent repetitive loading, providing specific portions of the cartilage an opportunity to be both mechanically stimulated and to recover.

\section{ESTIMATING ENERGETIC EXPENDITURE}

In Fig. 2 the cost of transport (normalized to mass) is depicted over a range of speeds for walking and running. Intermediate cost is provided by a simple addition of walk and run cost, which would correspond to an exercise session where half is spent walking and half running (i.e., with a single gait change adding negligible cost). Adding repeating gait switching increases cost, where 10-step intervals results in 7.5\% total increase and 5-step intervals in $15 \%$ increase. In the latter, cost is comparable to running even though half the activity involves walking.

A long-term training scenario is shown in Fig. 2 (arrows) where an individual begins training at relatively slow pace $(1 \mathrm{~m} / \mathrm{sec})$ with few gait switches (cost intermediate between walk and run at $1 \mathrm{~m} / \mathrm{sec}$ ). As the individual becomes more capable, the number of gait switches is increased and energetic cost increases (left vertical arrow). Once 5-step intervals are achieved treadmill speed can be increased. If this is too demanding a decrease in the rate of gait switching can bring the cost to whatever level is tolerable for the 
individual. Gait switching rate can be increased followed by another cycle of speed increase. Note that this plot indicates general cost and individuals will vary.

\section{Application to practice}

- Treadmill mini-intervals involving repeated walking, running and gait changes that is often adopted by individuals as they begin an activity program may have multiple benefits for increasing general fitness and functional capability.

- Combining these three separate activities will decrease the chance of repetitive overuse injury and can augment a training regime.

\section{CONCLUSIONS}

A mini-interval treadmill protocol incorporates three activities: running, walking, and gait switching. As such, it trains different coordination patterns that use a range of muscles in different ways. Switching increases metabolic demand, but through discrete events, which can be tolerated more easily by less fit individuals. It has the added benefit of developing stability during transitions while a safety hand rail is present, avoiding potential falls in more complex natural environments. Finally, increased mental focus may help avoid the monotony of usual treadmill workouts, aiding adherence to an exercise program.

\section{CONFLICT OF INTEREST}

No potential conflict of interest relevant to this article was reported.

\section{REFERENCES}

Abdolvahab M. Gait transition dynamics are modulated by concurrent cognitive activity. Atten Percept Psychophys 2015;77:2502-2506.

Alton F, Baldey L, Caplan S, Morrissey MC. A kinematic comparison of overground and treadmill walking. Clin Biomech (Bristol, Avon) 1998; 13:434-440.

Bertram JE, Hasaneini SJ. Neglected losses and key costs: tracking the energetics of walking and running. J Exp Biol 2013;216:933-938.

Cappellini G, Ivanenko YP, Poppele RE, Lacquaniti F. Motor patterns in human walking and running. J Neurophysiol 2006;95:3426-3437.

Cavagna GA, Thys H, Zamboni A. The sources of external work in level walking and running. J Physiol 1976;262:639-657.

Croft JL, Schroeder RT, Bertram JEA. The goal of locomotion: separating the fundamental task from the mechanisms that accomplish it. Psychon Bull Rev 2017;24:1675-1685.

Croft JL, Schroeder RT, Bertram JEA. The landscape of movement control in locomotion: cost, strategy, and solution. Front Psychol 2019;10:716.

Daniels GL, Newell KM. Attentional focus influences the walk-run transition in human locomotion. Biol Psychol 2003;63:163-178.

Diedrich FJ, Warren WH Jr. Why change gaits? Dynamics of the walk-run transition. J Exp Psychol Hum Percept Perform 1995;21:183-202.

Dromerick AW, Lum PS, Hidler J. Activity-based therapies. NeuroRx 2006; 3:428-438.

Duncan GE, Anton SD, Sydeman SJ, Newton RL Jr, Corsica JA, Durning PE, Ketterson TU, Martin AD, Limacher MC, Perri MG. Prescribing exercise at varied levels of intensity and frequency: a randomized trial. Arch Intern Med 2005;165:2362-2369.

Ganley KJ, Stock A, Herman RM, Santello M, Willis WT. Fuel oxidation at the walk-to-run-transition in humans. Metabolism 2011;60:609-616.

Hagio S, Fukuda M, Kouzaki M. Identification of muscle synergies associated with gait transition in humans. Front Hum Neurosci 2015;9:48.

Helgerud J, Høydal K, Wang E, Karlsen T, Berg P, Bjerkaas M, Simonsen T, Helgesen C, Hjorth N, Bach R, Hoff J. Aerobic high-intensity intervals improve $\mathrm{VO} 2 \mathrm{max}$ more than moderate training. Med Sci Sports Exerc 2007;39:665-671.

Hollman JH, Watkins MK, Imhoff AC, Braun CE, Akervik KA, Ness DK. A comparison of variability in spatiotemporal gait parameters between treadmill and overground walking conditions. Gait Posture 2016;43: 204-209.

Inman VT, Ralston HJ, Todd F. Human walking. Baltimore: Williams and Wilkins; 1981.

Kantak SS, Stinear JW, Buch ER, Cohen LG. Rewiring the brain: potential role of the premotor cortex in motor control, learning, and recovery of function following brain injury. Neurorehabil Neural Repair 2012;26: 282-292.

Kuo AD. The six determinants of gait and the inverted pendulum analogy: a dynamic walking perspective. Hum Mov Sci 2007;26:617-656.

Laursen PB, Jenkins DG. The scientific basis for high-intensity interval training: optimising training programmes and maximising performance in highly trained endurance athletes. Sports Med 2002;32:53-73.

Lee SJ, Hidler J. Biomechanics of overground vs. treadmill walking in healthy individuals. J Appl Physiol (1985) 2008;104:747-755.

Long LL 3rd, Srinivasan M. Walking, running, and resting under time, distance, and average speed constraints: optimality of walk-run-rest mixtures. J R Soc Interface 2013;10:20120980.

Pandy MG, Andriacchi TP. Muscle and joint function in human locomotion. Annu Rev Biomed Eng 2010;12:401-433.

Pires NJ, Lay BS, Rubenson J. Joint-level mechanics of the walk-to-run 
transition in humans. J Exp Biol 2014;217:3519-3527.

Prilutsky BI, Gregor RJ. Swing- and support-related muscle actions differentially trigger human walk-run and run-walk transitions. J Exp Biol 2001;204:2277-2287.

Saunders JB, Inman VT, eberhart HD. The major determinants in normal and pathological gait. J Bone Joint Surg Am 1953;35-A:543-558.

Segers V, De Smet K, Van Caekenberghe I, Aerts P, De Clercq D. Biomechanics of spontaneous overground walk-to-run transition. J Exp Biol 2013;216(Pt 16):3047-3054.

Segers V, Lenoir M, Aerts P, De Clercq D. Kinematics of the transition between walking and running when gradually changing speed. Gait Posture 2007;26:349-361.

Sentija D, Markovic G. The relationship between gait transition speed and the aerobic thresholds for walking and running. Int J Sports Med 2009; 30:795-801.

Srinivasan M. Fifteen observations on the structure of energy-minimizing gaits in many simple biped models. J R Soc Interface 2011;8:74-98.

Srinivasan M, Ruina A. Computer optimization of a minimal biped mod- el discovers walking and running. Nature 2006;439:72-75.

Tjønna AE, Lee SJ, Rognmo Ø, Stølen TO, Bye A, Haram PM, Loennechen JP, Al-Share QY, Skogvoll E, Slørdahl SA, Kemi OJ, Najjar SM, Wisløff

$\mathrm{U}$. Aerobic interval training versus continuous moderate exercise as a treatment for the metabolic syndrome: a pilot study. Circulation 2008; 118:346-354.

Usherwood JR. Why not walk faster? Biol Lett 2005;1:338-341.

Usherwood JR, Bertram JE. Gait transition cost in humans. Eur J Appl Physiol 2003;90:647-650.

White SC, Yack HJ, Tucker CA, Lin HY. Comparison of vertical ground reaction forces during overground and treadmill walking. Med Sci Sports Exerc 1998;30:1537-1542.

Wisløff U, Støylen A, Loennechen JP, Bruvold M, Rognmo Ø, Haram PM, Tjønna AE, Helgerud J, Slørdahl SA, Lee SJ, Videm V, Bye A, Smith GL, Najjar SM, Ellingsen Ø, Skjaerpe T. Superior cardiovascular effect of aerobic interval training versus moderate continuous training in heart failure patients: a randomized study. Circulation 2007;115:30863094. 